\title{
Gamma-rays from comptonization of stellar radiation in the binary system containing PSR J2032+4127 at its periastron passage
}

\author{
Wlodek Bednarek* \\ Department of Astrophysics, University of Lodz, Lodz, Poland \\ E-mail: bednareuni.lodz.pl \\ Piotr Banasinski \\ Department of Astrophysics, University of Lodz, Lodz, Poland \\ E-mail: p.banasinski@uni.lodz.pl
}

\begin{abstract}
We calculate the high energy gamma-ray emission expected during the periastron passage of the pulsar PSR J2032+4127 which form extended binary system with the massive companion star MT91 213. The $\gamma$-ray emission is expected due to the comptonization of the stellar radiation by electrons accelerated at the pulsar wind and stellar wind collision shock. We apply the numerical code for the cascade process initiated by electrons in the anisotropic radiation from the companion star. We conclude that around the periastron passage of this binary system, the $\gamma$-ray emission at $\mathrm{TeV}$ energies can overcome the $\mathrm{TeV}$ emission observed from an extended source in this direction which is likely due to the pulsar wind nebula formed by the pulsar PSR J2032+4127. We discuss the conditions within the binary system for which such a point like $\mathrm{TeV} \gamma$-ray source should appear in addition to the presently observed the extended $\mathrm{TeV} \gamma$-ray source.
\end{abstract}

35th International Cosmic Ray Conference - ICRC2017-

10-20 July, 2017

Bexco, Busan, Korea

\footnotetext{
* Speaker.
} 


\section{Introduction}

High energy $\gamma$-ray emission from only one binary system, containing radio pulsar PSR B125963 and Be type massive companion SS2883, has been observed and studied in more detail at TeV energies [1, 目, 3]. Another young, radio pulsar, PSR J2032+4127, has been proposed to be a companion of the massive Be type star, MT91 213, which parameters are similar to the star SS2883 [П]. This binary system is much more extended than PSR B1259-63/SS2883. It is expected that PSR $\mathrm{J} 2032+4127$ passes the periastron late in 2017 [阿]. The high energy X-ray and $\mathrm{GeV} \gamma$-ray emission from this binary has been recently studied in [6]. Those authors discuss the Inverse Compton (IC) $\gamma$-ray emission in the context of the wind collision model for the massive binary systems. The observations of the binary system PSR J2032+4127/MT91 213 in the TeV $\gamma$-ray energy range are expected to be more difficult since in the direction of this binary an extended, steady $\mathrm{TeV} \gamma$-ray source (TeV J2032+4130) has been discovered [ד] (and confirmed in [8, 9]). Applying the available parameters of the binary system, PSR J2032+4127/MT91 213, we present detailed calculations of the $\mathrm{GeV}-\mathrm{TeV} \gamma$-ray radiation expected from this binary close to the periastron passage of the pulsar. For this purpose, we use the Monte Carlo cascade code which follows production of $\gamma$-rays by relativistic electrons, accelerated in the wind collision region, which comptonize well defined radiation field from the companion star.

\section{PSR J2032+4127/MT91 213 system}

The $\gamma$-ray pulsar PSR J2032+4127, with the period of $P=143 \mathrm{~ms}$, has been discovered by the Fermi-LAT [10]. The spin down power of this pulsar is $L_{\mathrm{PSR}}=2.7 \times 10^{35} \mathrm{erg} \mathrm{s}^{-1}$, the characteristic age is $115.8 \mathrm{kyr}$, the surface magnetic field is estimated on $B_{\mathrm{PSR}}=1.7 \times 10^{12} \mathrm{Gs}$ [ [10], and the distance $\mathrm{d}=1.33 \pm 0.06 \mathrm{kpc}$ [11]. The pulsar is on the elongated orbit around the companion star, MT91 213, which is a B0 V type star with the luminosity $L_{\star}=5.8 \times 10^{37} \mathrm{erg} \mathrm{s}^{-1}$ and the effective surface temperature $3.1 \times 10^{4} \mathrm{~K}[12]$. The radius of this star is $R_{\star}=3 \times 10^{11} \mathrm{~cm}$.

The basic parameters of the pulsar in the binary system are not precisely known since only a part of the pulsar orbit has been observed up to now. According to the model 2 of Ho [5], the semi-major axis of the binary system is $a=2.7 \times 10^{14} \mathrm{~cm}$, and the eccentricity is $e=0.961$. For these parameters, the distance of the pulsar from the companion star during the periastron passage is $D_{\text {peri }}=a(1-e) \approx 1.05 \times 10^{13} \mathrm{~cm}$, and the distance between the stars in the superior conjunction of the pulsar is $D_{\text {sup }}=a\left(1-e^{2}\right) /\left(1+e \cos \left(90^{\circ}+\omega\right) \approx 1.28 \times 10^{13} \mathrm{~cm}\right.$, where the longitude of the periastron is $\omega=40^{\circ}$ (for the model 2 in [5]). The angle between the line of sight and the direction defined by the centers of the stars is $\cos \left(180^{\circ}-\alpha\right)=\cos \left(90^{\circ}+\omega\right) \cos \left(90^{\circ}-i\right)$, where $i$ is the inclination angle of the binary system (see Fig. 1).

The pulsar PSR J2032+4127 is expected to form a pulsar wind which interaction with the wind of the companion star can be quite complicated. Close to the periastron, the winds create a collision region, between the star and the pulsar, in which particles can be effectively accelerated to energies allowing them production of the X-ray radiation in the synchrotron process and also the TeV $\gamma$-rays in the IC scattering of the stellar radiation [13, 14, 15, 16]. The fate of the pulsar wind after collision with the stellar wind is not well known. Two limiting cases can be imagined: the collision of the ideal homogeneous winds and the collision of the very turbulent winds. If the 


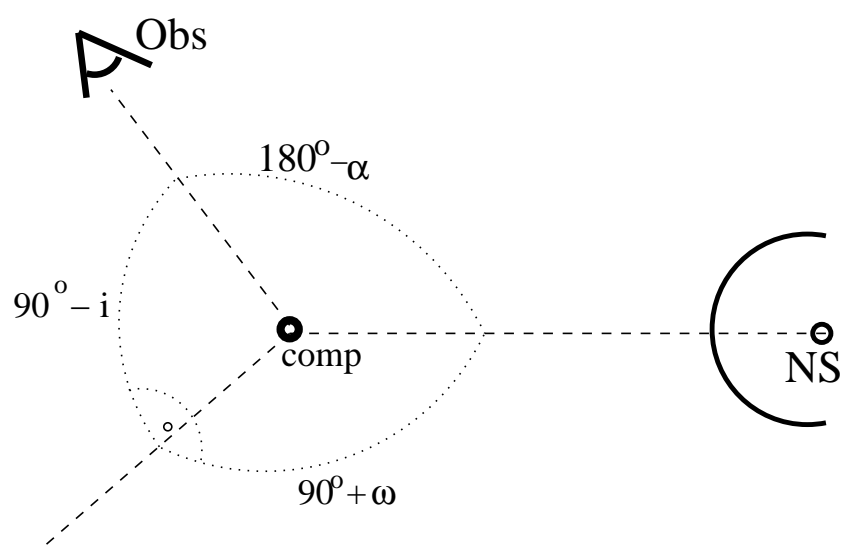

Figure 1: Schematic representation of the geometry of the binary system containing PSR J2032+4127 at the periastron passage. The pulsar is surrounded by the wind collision region (thick solid curve), in which electrons are accelerated. The observer sees the companion star at the angle $\alpha$, which is measured between the directions defined by the centers of the companion star "comp" and the pulsar "NS" and the direction towards the observer "Obs". The inclination angle of the binary system is $i$ and the longitude of the periastron passage of the pulsar is $\omega$.

winds are homogeneous, then the winds after the shocks are expected to form a separate fluids. The pulsar wind can even accelerate along the shock structure as a result of the adiabatic expansion [17, 18, 19]. In the second model, the very in-homogeneous, turbulent stellar wind loads the pulsar wind with the barionic matter [20, 21, 22]. In the limiting case, when the winds mix effectively the pulsar wind can be significantly decelerated to velocity $v_{\text {mix }}$. To estimate the order of magnitude of the velocity of the winds after mixing we can make use of the idealistic scenarios in which the colliding winds conserve their energy and momentum. The conservation of the energy gives us the upper limit on the velocity of the mixed winds since a part of the energy of the winds is expected to be thermalised in the collision process. In this case, the mixed wind velocity can be estimated as shown in [20] (see Eq. 1 and description below). In this limit the velocities of the mixed winds can be of the order of a few to several percent of the velocity of the light. On the other hand, the conservation of momentum of the colliding winds (but not very turbulent) gives the lower limit on the mixed wind velocity [23]. In this case, the mixed wind velocity can change from close to zero at the apex and increase up to the velocity of the stellar wind (before mixing) at regions along the collision region which are far away from the apex. In our calculations we use the range of velocities which are laying between those two limits. Note that in the case of efficient mixing, electrons accelerated in the turbulent winds are relatively slowly advected from the vicinity of the companion star, allowing for more efficient comptonization of the stellar radiation to the $\gamma$-ray energy range.

The distance, between the apex of the wind collision region and the pulsar, can be estimated from, $R_{\mathrm{sh} / \mathrm{PSR}}=D \sqrt{\eta} /(1+\sqrt{\eta})$, where $D=10^{13} D_{13} \mathrm{~cm}$ is the separation of the objects, $\eta=$ $L_{\mathrm{PSR}} /\left(c \dot{M}_{\star} v_{\star}\right) \approx 0.14 / M_{-8} v_{8}$ is the ratio of the pressures of the winds from the pulsar and the companion star [24], $\dot{M}_{\star}=10^{-8} M_{-8} \mathrm{M}_{\odot} \mathrm{yr}^{-1}$ and $v_{\star}=10^{8} v_{8} \mathrm{~cm} \mathrm{~s}^{-1}$ are the mass loss rate and the velocity of the companion star wind, $L_{\mathrm{PSR}}=10^{35} L_{35} \mathrm{erg} \mathrm{s}^{-1}$ is the pulsar power, and $c$ is the velocity of light. 


\section{Gamma-rays from IC pair cascade}

We assume that electrons can be accelerated in the magnetized, turbulent collision region of the pulsar and stellar winds. Their acceleration time scale can be defined by the acceleration coefficient $\xi$ and the magnetic field strength in the acceleration region (e.g. see Eq. 5 in [20]). Electrons are also advected with some velocity $v_{\text {mix }}$ from the acceleration site. The characteristic time scale for the advection process can be estimated from, $\tau_{\mathrm{adv}} \approx R_{\mathrm{sh} / \mathrm{PSR}} / v_{\text {mix }} \approx$ $10^{4} D_{13} \sqrt{\eta} /(1+\sqrt{\eta}) / v_{9} \mathrm{~s}$, where $v_{\text {mix }}=10^{9} v_{9} \mathrm{~cm} \mathrm{~s}^{-1}$. The advection time scale of electrons limits their acceleration process to the maximum possible energies, $E_{\max }^{\mathrm{adv}} \approx 110 \sigma_{-1}^{1 / 2} \xi_{-2} / v_{9} \mathrm{TeV}$. where $\xi=0.01 \xi_{-2}$ is the acceleration coefficient, and $\sigma=0.1 \sigma_{-1}$ is the magnetization parameter of the pulsar wind. The value of $\xi$ can be related to the velocity of the mixed winds in the following way $\xi \sim\left(v_{\operatorname{mix}} / c\right)^{2}$, if particles are energized after the mixing process of the winds. However, if the acceleration process of electrons occurs already in the region of the pulsar wind then, $\xi$ can be fixed to $\sim 0.1$ since the pulsar wind velocity slows down to $\sim 0.3 \mathrm{c}$. The acceleration process can be saturated by the electron's energy losses on the synchrotron process (the cooling time scale is given by Eq. 8 in [20]). Then the maximum energies of electrons are limited to, $E_{\max }^{\mathrm{syn}} \approx 6.1\left(\xi_{-2} / B_{\mathrm{sh}}\right)^{1 / 2} \approx 17.5\left[\xi_{-2} \sqrt{\eta} D_{13} / \sigma_{-1}^{1 / 2}(1+\sqrt{\eta})\right]^{1 / 2} \mathrm{TeV}$. The magnetic field at the winds collision region is estimated by the simple extrapolation from the vicinity of the pulsar, $B_{\mathrm{Sh}} \approx\left(B_{\mathrm{PSR}}\left(R_{\mathrm{NS}} / R_{\mathrm{LC}}\right)^{3}\left(R_{\mathrm{LC}} / R_{\mathrm{sh}}\right) \sigma^{1 / 2} \approx 0.11 \sigma_{-1}^{1 / 2}(1+\sqrt{\eta}) / \sqrt{\eta} D_{13} \mathrm{G}\right.$, where $R_{\mathrm{NS}}=10^{6} \mathrm{~cm}$ is the radius of the pulsar, $R_{\mathrm{LC}}=c P / 2 \pi \approx 6.8 \times 10^{8} \mathrm{~cm}$ is the light cylinder radius of the pulsar.

We assume that electrons are accelerated at the distance of the apex of the collision region formed in collisions of stellar and pulsar winds. They obtain a power law spectrum with the spectral index equal to -2 up to the maximum energy determined above. It is assumed that relativistic electrons can take up to $10 \%$ of the spin down power of the pulsar. The angular distribution of primary electrons is isotropic in the plasma reference frame. Electrons are slowly advected from the acceleration place on the advection time scale. During this escape process, electrons lose energy on the synchrotron radiation and on the IC scattering of the anisotropic radiation from the companion star. We calculate the synchrotron spectrum which is emitted isotropically by the electrons since the magnetic field is assumed to be random in the reference frame of the plasma. In contrast, $\gamma$-rays in the IC process are produced anisotropically due to the anisotropic radiation of the companion star as seen from the injection place of electrons. $\gamma$-rays, produced in the general direction towards the companion star, are additionally absorbed in the stellar radiation. As a result of the absorption of primary $\gamma$-rays, the anisotropic IC $e^{ \pm}$pair cascade develops in the surrounding of the massive star. In order to calculate the $\gamma$-ray spectra which emerge from the binary system at specific directions, we apply the specific IC $e^{ \pm}$pair cascade model in which the secondary $e^{ \pm}$pairs are isotropised by the random component of the magnetic field close to their place of creation (at first such model has been discussed in [25, 26]).

\section{TeV $\gamma$-rays from the binary containing PSR 2032+4127}

The HEGRA TeV $\gamma$-ray source ( TeV J2032+4130 [ך]) has the extension of $\sim 6$ ', and the flux of $5 \%$ of the Crab Nebula [29, 8, 9, 30, 31]. The nature of the HEGRA TeV source remains unclear. The discovery of the energetic pulsar in this direction, PSR 2032+4127, seems to support 

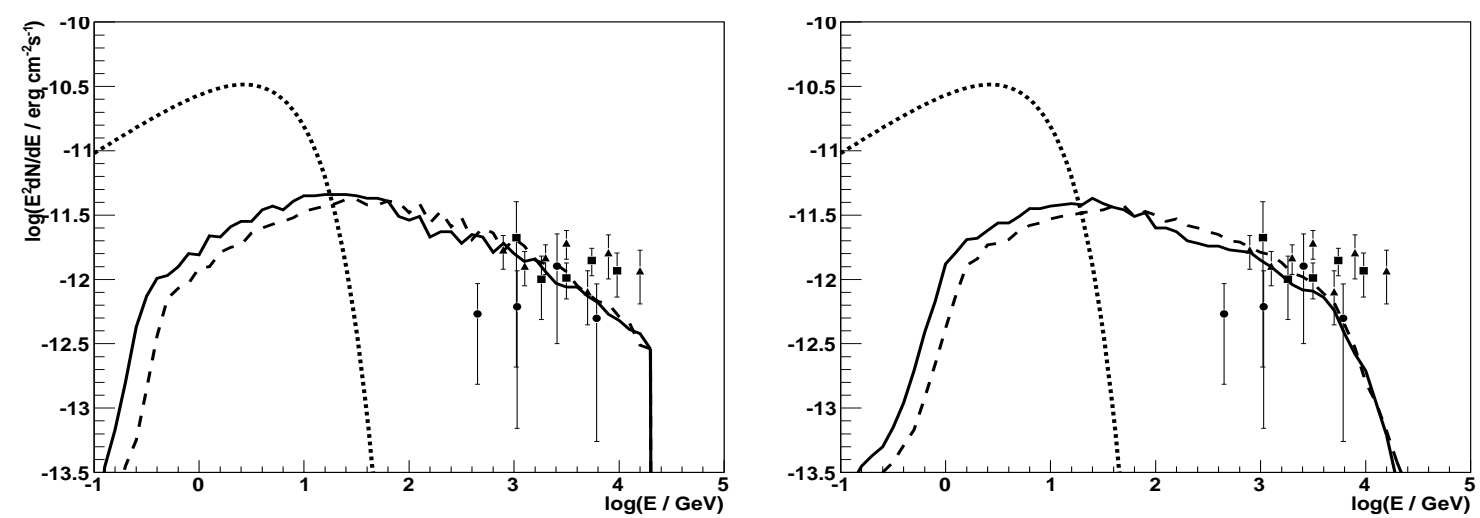

Figure 2: The IC spectra (SED), at the periastron passage are compared with the TeV $\gamma$-ray spectrum of the extended source in the location of PSR 2032+4227, measured by the HEGRA (black squares [D]), MAGIC (circles [8]), and VERITAS (triangles [9]). Figure on the left is for $\eta=1, \xi=0.1, v_{\text {mix }}=7 \times 10^{9} \mathrm{~cm} \mathrm{~s}^{-1}$ and $\sigma=0.003$; and on the right for $\eta=1, \xi=0.1, v_{\text {mix }}=7 \times 10^{9} \mathrm{~cm} \mathrm{~s}^{-1}$ and $\sigma=0.1$. The spectra are calculated for the inclination of the binary system equal to $i=60^{\circ}$ (solid curves) and $i=0^{\circ}$ (dashed curves). The pulsed $\gamma$-ray spectrum of PSR 2032+4127 [5] is marked by the dotted curve.

the pulsar wind nebula hypothesis [32, 33]. The IC scattering of stellar radiation can give important contribution when the pulsar is close to its periastron. In order to determine the level of the emission from the binary system to already observed $\mathrm{TeV} \gamma$-ray emission from the nebula, we confront the $\mathrm{TeV} \gamma$-ray spectrum from the nebula around the pulsar with the $\mathrm{TeV} \gamma$-ray emission due to the comptonization of stellar radiation by electrons accelerated at the collision region. In Fig. 2, we show the $\gamma$-ray spectra calculated for the pulsar at the periastron (the strongest radiation field of the companion star), for specific range of parameters describing the process of the acceleration and the interaction of electrons with stellar radiation. The spectra are calculated for the wide range of the inclination angles of the binary system $\left(i=0^{\circ}\right.$ and $\left.60^{\circ}\right)$. The contribution to the TeV $\gamma$-ray emission from the binary system to the extended $\mathrm{TeV}$ emission from the nebula becomes important when the parameter $\eta$ is not very far from unity (see Fig. 2). Our present calculations show that at the periastron passage, the $\mathrm{TeV}$ emission from the binary system PSR 2032+4127 can dominate over the emission from the PWNa but only at energies lower than a few TeV. Electrons with larger energies are not able to scatter efficiently stellar radiation due to the Klein-Nishina effects and also their dominant synchrotron energy losses. The features of the $\mathrm{TeV} \gamma$-ray emission, for the pulsar located at the superior conjunction, are quite similar to those observed at the periastron (but on a slightly lower level). Both these locations are separated by a few weeks. Therefore, the TeV $\gamma$-ray emission from the binary system between periastron and superior conjunction is expected to be on the highest level. A few weeks time scale observations should allow detection of such an enhanced point-like emission by presently operating Cherenkov telescopes such as MAGIC and VERITAS. Outside the region between the periastron and the superior conjunction, the pulsar (and relativistic electrons) stays at clearly larger distance from the companion star and/or they are located more in the front of the companion star. Such geometrical situation is not favored for the efficient production of $\gamma$-rays by scattering stellar radiation. 


\section{Conclusion}

We have presented the results of calculations of the $\gamma$-ray emission from the anisotropic IC $e^{ \pm}$ pair cascades initiated by relativistic electrons in the vicinity of the massive star within the binary system containing PSR 2032+4127. We considered only well defined background radiation field within the binary system which is provided by the surface of the companion star. Therefore, calculated by us IC $\gamma$-ray spectra from the binary system PSR $2032+2741$ should be considered as the lower limits especially above $\mathrm{TeV}$ energies. We show that $\gamma$-ray emission from the binary system can overcome the level of the $\mathrm{TeV}$ emission from the HEGRA source provided that electrons are relatively slowly advected from the vicinity of the massive star. Such situation is expected when the stellar and pulsar winds mix effectively in the wind collision region and the velocity of the stellar wind is not very large (i.e. $v_{\star} \leq 10^{8} \mathrm{~cm} \mathrm{~s}^{-1}$ ). Moreover, the collision region should be close to the massive star. This happens when the pressure of the stellar wind does not dominate completely over the pressure of the pulsar wind, i.e. the parameter $\eta$ describing the wind collision region is not far from unity. For $\eta \sim 1$, the collision region of the winds is far from the pulsar and relatively close to the stellar surface. Then, the magnetic field in the mixed pulsar wind is relatively weak (due to the larger distance from the pulsar). Therefore, the energy losses of relativistic electrons on the synchrotron process are reduced and energy losses on the IC process are enhanced. The IC $\gamma$-ray fluxes, shown in Figs. 4, are expected to be clearly larger if the mixed wind velocity is below the value considered in this figure. However, the efficiency of electron acceleration can be lower than assumed $10 \%$ since only a part of the wind regions can provide energy for the acceleration process. Therefore, comparison of our calculations with the future observations of the binary system PSR 2032+2741/MT91 213 during periastron can allow to constrain the range of allowed values for parameters, determining the geometry of the collision region $\eta$, efficiency of electron acceleration and the velocity of the mixed winds. Note that, these parameters can be also constrained by the independent observations in other energy ranges. Then, such constraints will allow determination of the acceleration efficiency of electrons with this binary system based on the $\mathrm{TeV}$ $\gamma$-ray observations and modeling of the type presented here.

Our calculations show that the conditions for the $\gamma$-ray production within the binary system PSR 2032+4127 close to the periastron are less favorite than those in the case of the binary system PSR B1259-63/SS2883. This is generally expected since the energy loss rate of the pulsar PSR $2032+2741$ is a factor of about 3 lower than the pulsar PSR B1259-63. Closer location of the pulsar PSR B1259-63 to the companion star at the periastron and its larger energy loss rate moves the wind collision region, and so the place of electron acceleration, is closer to the companion star than in the case of the binary system containing PSR 2032+2741. Detailed observations of the binary system PSR 2032+2741 by the Cherenkov telescopes close to the periastron, linked with the future multi-wavelength studies of the stellar wind from the companion star, should provide interesting independent constraints on proprieties of the pulsar winds, conditions for the acceleration of particles, and the details of their radiation mechanisms. At present, such detailed comparison of these two binary systems is not possible due to the lack of knowledge on the possible contribution of additional radiation fields and their geometry (e.g. stellar disk) in the binary PSR 2032+4127.

\section{Acknowledgements}

This work is supported by the grant through the Polish Narodowe Centrum Nauki No. 2014/15/B/ST9/04043 


\section{References}

[1] F. Aharonian Astron.Astrophys. 442 (2005) 1

[2] F. Aharonian et al. Astron.Astrophys. 507 (2009) 389

[3] A. Abramowski et al. Astron.Astrophys. 551 (2013) A94

[4] A.G. Lyne et al. Mon.Not.R.Astr.Soc. 451 (2015) 581

[5] W.C.G. Ho et al. Mon.Not.R.Astr.Soc. 464 (2017) 1211

[6] J. Takata et al. Astrophys. J. 836 (2017) 241

[7] F. Aharonian et al. Astron.Astrophys. 393 (2002) L37

[8] J. Albert et al. Astrophys. J. 675 (2008) L25

[9] E. Aliu Astrophys. J. 783 (2014) 16

[10] A.A. Abdo et al. Science 325 (2009) 840

[11] D.C. Kiminki et al. Astrophys. J. 811 (2015) 85

[12] N.J. Wright, J.E. Drew, M. Mohr-Smith Mon.Not.R.Astr.Soc. 449 (2015) 741

[13] L. Maraschi, A. Treves Mon.Not.R.Astr.Soc. 194 (1981) 1

[14] M. Tavani, J. Arons, V.M. Kaspi Astrophys. J. 433 (1994) L37

[15] M. Tavani, J. Arons Astrophys. J. 477 (1997) 439

[16] J.G. Kirk, L. Ball, O. Skjaeraasen Astropart.Phys. 10 (1999) 31

[17] S.V. Bogovalov et al. Mon.Not.R.Astr.Soc. 387 (2008) 63

[18] G. Dubus, B., Cerutti, G., Henri Astron.Astrophys. 516 (2010) 18D

[19] S.W. Kong, K.S. Cheng, Y.F. Huang Astrophys. J. 753 (2012) 127

[20] W. Bednarek Astron.Astrophys. 561 (2014) A116

[21] X. Paredes-Fortuny et al. Astron.Astrophys. 574 (2015) 77

[22] V.M. de la Cita et al. Astron.Astrophys. 598 (2017) A13

[23] J. Canto, A.C. Raga, F.P. Wilkin Astrophys.J. 469 (1996) 729

[24] T. Girard, L.A. Wilson Astron.Astrophys. 183 (1987) 247

[25] W. Bednarek Astron.Astrophys. 322 (1997) 523

[26] W. Bednarek Astron.Astrophys. 362 (2000) 646

[27] C.F. Kennel, F.V. Coroniti Astrophys. J. 283 (1984) 710

[28] R.R. Sefako, O.C. de Jager Astrophys. J. 593 (2003) 1013

[29] F. Aharonian et al. 2005 Astron.Astrophys. 431 (2005) 197

[30] M.J. Lang et al. Astron.Astrophys. 423 (2004) 415

[31] A. Konopelko et al. Astrophys. J. 658 (2007) 1062

[32] W. Bednarek, W. Mon.Not.R.Astr.Soc. 345 (2003) 847

[33] H. Murakami et al. Pub.Astron.Soc.Pacific 63 (2011) 873 